\title{
AUTOMATION IN BUILDING DESIGN WITH SPATIAL INFORMATION
}

\author{
Tang-Hung Nguyen \\ Assistant Professor, Department of Civil Engineering, North Dakota State University, \\ 120D CME Building, Fargo, ND 58105 - Tel.: (701) 231-8646 - Email address: \\ hung.nguyen@ndsu.nodak.edu.
}

\section{Amr A. Oloufa}

Associate Professor and Director of Construction Engineering Program, Department of Civil and Environmental Engineering, University of Central Florida, P.O. Box 16245, Orlando, FL 32816 - Tel.: (407) 823-3592 - Email address: aoloufa@mail.ucf.edu

\begin{abstract}
While current CAD (Computer-Aided Design) systems provide a variety of data representation schemes (e.g. wire-frames, surface, and solid modeling) and data exchange protocols, they fail to address automation issues in retrieval of building information to be used in different AEC (Architecture, Engineering, and Construction) applications. In these CAD systems, topological information (also known as spatial relations) describing the threedimensional spatial relationships between building components is conventionally represented in a manual fashion into data models. The manual data representation, however, inherently is a complex and challenging task due to the wide variety of spatial relationships. This paper outlines a computer-based building design framework with emphasis on the engine capable of automatically deducing topological information of building components, which support various aspects in building design such as constructability analysis, construction planning, and building code compliance checking.
\end{abstract}

Keywords: Computer-Aided Design, Topological Information, Automated Building Design Systems, Solid Modeling.

\section{Introduction}

Presently, most CAD systems provide two major tools for representing data of building components: the geometric modeling system dealing with spatial abstractions and the database management system dealing with functional information. The spatial information describes the building components' geometry ((i.e. dimensions and locations) and topology (i.e. spatial relations among the components), while the functional data represents all the other discipline-specific properties of the components (e.g. structural, thermal properties, design constraints, and building regulations). Formal representation of spatial data in general and topological data in particular of building components is a complex and challenging task in developing building design systems. The complexity is in part due to the fact that each professional usually utilizes his/her own representation of topologies and dimensionalities to express spatial information of building components. Furthermore, different design tasks require different types of topological information. For example, information about the walls surrounding a particular space (e.g. fire zone) is needed for code compliance checking of that space, details of connections between individual structural members of a reinforced concrete frame should be provided for reasoning about constructability, and information about the adjacency among floors of a high-rise building is required for planning the sequence of construction activities. The variety of topological information to be used throughout the design and construction process may contribute to complexity in data definition, representation and retrieval. Moreover, the topological information of building/product elements is conventionally structured directly in product data models, in which designers are prompted to manually specify spatial information of interest to different disciplines. Such a manual representation is usually subject to data inconsistency, incompleteness, and prone to error, 
which may result in a misinterpretation of information. Thus, the topological information should be classified and modeled in such a way that the required spatial data for a particular design task (e.g. energy analysis, building code compliance checking, constructability reasoning, and construction planning) can be automatically retrieved. This paper attempts to identify and classifies various topological information commonly used in AEC disciplines into more specific categories such as adjacency, connection, containment, separation, and intersection. These topological relations are then incorporated into a CAD system so that the required spatial relationships between building components can be automatically deduced to support different design tasks.

\section{Classification of Topological Information}

The first step taken in the development of the proposed building design system is to identify various topological information (i.e. spatial relations between building components) commonly used in the AEC domain. The spatial relations are then classified into several main categories to support automatic deduction of building information required by different project participants. Five main categories of the topological information have been identified and named separation, adjacency, connectivity, intersection, and containment.

Separation: refers to the relation where two building components are physically separate from each other. Information about the separation relation of a building component with others can be used to check for its code compliance. For example, "Individual roof panels shall be separated from each other by a distance of not less than 4 feet measured in a horizontal plane" [BOCA Subsection 2607.2, 1993], implies the requirement of a separation between two roof panels.
Adjacency: is introduced to express the type of vicinity that may exist between building components. Depending on the location of one component relative to another one adjacent-to it, the vicinity relations may be represented by different terms, such as next-to (e.g. mechanical room is next-to electrical room), above, below (e.g. parking space is below lobby area), righthand-side, and left-hand-side.

Connectivity: relates to modeling connected-to, attached-to or supported-by relations between building components. For examples, a suspended acoustical tile ceiling is connected-to a concrete frame, a rigid insulation is attached-to a protected roof membrane, and a beam is supported-by a column.

- Containment: corresponds to contained-in or included-in relations. This type of topological information can describe the spatial relationships between two spaces (e.g. an incinerator is contained-in a room space), two building components (e.g. a window is contained-in a wall), or between a building component and a space (e.g. an air duct is contained-in a ceiling space or plenum).

- Intersection: represents shared-by relations. A typical application of this type of topological information is that the intersection between a tower crane's boom and an existing building helps verify for constructability of a new building where the available tower crane is selected for material delivery.

The classification of topological relations provides a basis for establishing a building data structure facilitating the organization and management of various topological relations in a building data model. Such a data structure is helpful in making it easy to develop algorithms responsible for the automated deduction of spatial information in the proposed computer-based building design system.

\section{The Automated Building Design System}


Basically, the proposed computerbased building design system is built on top of a 3D (three-dimensional) solid modeling system and is capable of automatically deducing various topological relationships between building design objects (e.g. beams, columns, windows, walls, etc.). To achieve such an automated design system, the algorithms for deduction of the five main categories of topological relations described above must be developed beforehand.

\section{Deduction Algorithms}

As the proposed building design system is developed in a 3D solid modeler, such solid primitives as vertex, edge, face, cell, and loop are used to describe each building objects. The geometric and topological information about theses primitives can be extracted from the $3 \mathrm{D}$ CAD system to deduce the spatial relationships between building objects. Basically, the relationships between vertices and faces representing building components determine spatial relationships between those components. There are three possible relationships between a vertex and a face, i.e. the vertex could lies on the face, to the right or above, or to the left or below the face. This leads to the need for a definition of face outward normal vectors. For this study, all face outward normal vectors are defined by the right hand rule which takes the list of vertices to be counter-clockwise and specifies the outward normal to be the one giving the positive value for points outside the solid objects, as shown in Figure 1.

\section{Figure 1. Convention for Face Outward Normal}

The building element to be considered for illustrating the deduction of topological information in the proposed system is the space. Depending on the position of a vertex with respect to a face, the value representing the vertex-face relationships can be assigned to $-1,1$, and 0 , which indicates that the vertex is to the negative side of the face, that is, inside the building space; 1 indicates that the vertex is to the positive side of the face, thus outside the space; and 0 represents those vertices that lie on the face itself.

Example: The algorithm identifies spaces that are adjacent-to, i.e. next-to, above, and below, a given space. In other words, the algorithm determines whether the given space shares a common face with any other spaces and all vertices other than those defining the common face are outside the given space. The development of this algorithm is based on several assumptions as follows.

1. All outward normal vectors of faces are defined by the right hand side rule which takes the list of vertices to be counter-clockwise and specifies the outward normal to be the one giving a positive value for points outside the space.

2. All faces bounding a building space are planar and defined by the following equation:

$$
\mathrm{Ax}+\mathrm{By}+\mathrm{Cz}+\mathrm{D}=0
$$

3. The values obtained by the substitution of the coordinates of the vertices in the equation of the faces, i.e. Relation Indexes (RI), could be positive, negative, or zero, indicating that the vertex is outside, inside, or on the space respectively.

4. All faces comprising a building space are assumed to be convex. It is noticed that the configuration of a building space can be either concave or convex, which is a critical issue. A simple check can be made to differentiate between the two: if a space has any face that gives a point inside the space with a positive $\mathrm{RI}$, the space is concave; if there is no such a face in the space, it is convex. For simplicity, the building spaces being considered in this work are assumed to be convex.

- Input: S1, S2, and S3 as spaces of a building where $\mathrm{S} 1 \neq \mathrm{S} 2 \neq \mathrm{S} 3$ (see Figure 2)

- Output: $\mathrm{S} 1$ is next-to $\mathrm{S} 2$ and below $\mathrm{S} 3$

- Algorithm:

1. For every vertex of S2 (or S3) and every face of $\mathrm{S} 1$, determine vertices of S2 that lie on a face of S1 (i.e. vertices that give zero RI values with respect to the face). These vertices 
define a common face between S1 and S2 (or S3).

2. For vertices other than those of the common face of S2 (or S3), compute the RI's indicating their positions with respect to $\mathrm{S} 1$.

3. If the RI is positive and the outward normal vector of the common face is parallel to XY plane, then S2 is next-to S1. In the case of $\mathrm{S} 3$ that also shares a common face with $\mathrm{S} 1$ and the outward normal vector of the common face is perpendicular to the $\mathrm{XY}$ plane, then $\mathrm{S} 1$ is below $\mathrm{S} 3$ or $\mathrm{S} 3$ is above $\mathrm{S} 1$.

\section{Figure 2. 3D Models of Building Objects}

For more elaborate examples and algorithms to deduce other categories of topological relations, the reader may refer to [Nguyen, 1999].

The Proposed Building Design System: TopoInfo

The algorithms for deducing the five categories of topological information (i.e. adjacency, containment, intersection, separation, and connectivity) have been implemented into a computer-based building design system, named TopoInfo. TopoInfo is developed on top of AutoCAD Release 14 that provides the underlying geometric solid modeler, in which building components such as columns, beams, slabs, windows, doors, etc. are described as 3D solids. Once the building components have been inputted as 3D solid objects, the basic data including geometry and topology about their primitive representing elements (i.e. vertices, edges, faces, and loops) are created and stored in AutoCAD database. These basic data then can be extracted by means of deduction mechanisms and algorithms to deduce spatial relationships between the building components. This can be done by executing a new command, named CheckTopo, which has been developed using an AutoCAD application development tool (e.g. ObjectARX) and added to the AutoCAD system. The process of the deduction can be summarized as follows:

First, from the AutoCAD drawing editor, the user is prompted to input geometric data and dimension (X-side, Y-side, Z-side, and location) describing the desired 3D building objects. The AutoCAD interface provides high-level and precise mechanisms for manipulating various types of geometric data structures and high-level geometric modeling objects such as prism, cylinder, polygon, line, etc. for creating basic design objects such as columns, beams, slabs, walls ... as shown in Figure 3. These basic objects can be "assembled" to create more complex building objects such as rooms, floors, or construction zones. At the lowest-level data structures, the basic objects are represented as $3 \mathrm{D}$ solids by means of primitive elements such as vertices, edges, and faces whose basic information are stored in AutoCAD database. Then, a new AutoCAD command (namely CheckTopo) that is added into the TopoInfo system is called to extract the basic geometric data from the AutoCAD database and check for topological relations between the building objects. This command basically contains a number of functions or methods being developed based on the deduction algorithms described previously. These functions are implemented into the TopoInfo system as a deduction engine, which is responsible for extracting basic 3D geometric information of building objects and deducing spatial relations between these objects. Finally, the output including the deduced topological relations can be stored and retrieved, when needed, to support different tasks of building design such as construction planning, constructability reasoning, and code compliance checking. The output can also be displayed in AutoCAD screen providing designers with a quick feedback on spatial relations (e.g. interference, conflicts) among building components.

Figure 3. 3D Model of a Typical Building 


\section{Applications of Topological Information}

Information about topological relations is essential to different AEC professionals throughout the project life cycle, spanning from building design to construction planning and maintenance. Examples of the applications of topological information to building design and construction, in general, include the following:

- Structural engineers require information about connections between individual structural elements for structural performance analysis or constructability evaluation,

- Architects are more concerned about the adjacency and intersection between building spaces and their boundaries for creation of building shapes and space layouts,

- Building inspectors need information (e.g. thermal properties) of the surrounding walls of a particular type of space for building code compliance checking.

- Lighting designers need information (e.g. glazing areas) of windows and/or doors contained in exterior walls for day lighting load calculations.

- Construction planners need information about the vicinity among construction zones for determining the interdependencies of construction activities to be performed in various spaces, and

- HVAC engineers use topological relations between spaces and their enclosing structures for heat-loss calculations and thermal analysis.

\section{Conclusion}

Various types of spatial or topological relationships among building components have been identified and classified into five main categories, i.e. adjacency, containment, intersection, separation, and connectivity which are essential building information to be repeatedly shared and reused by project participants throughout the design and construction process. This classification is helpful in facilitating the grouping and management of various topological relations in a computerized building data model. The implementation of the proposed building design system, namely TopoInfo, demonstrates the feasibility and practicality of using 3D solid modeling environment to develop a building design system capable of automatically generating complex building information such as spatial relations among building components, which can be retrieved to support different building design activities such as construction planning, constructability reasoning, and code compliance checking.

\section{References}

Augenbroe, G.: "COMBINE II: Computer Models for the Building Industry in Europe", Final Report, Technische Universiteit, Delft, Netherlands, 1995.

Björk, B.: "The Topology of Spaces, Space Boundaries and Enclosing Structures in Building Product Data Models", the CIB W78 Workshop on Computer-Integrated Construction, Montreal, Canada, 56-92, 1992.

BOCA: "The BOCA (Building Officials and Code Administrators) National Building Code", 12 $2^{\text {th }}$ Edition, Building Officials and Code Administrators International, Inc., Country Club Hills, IL, U.S.A, 1993.

Fischer, M.: "Automating Constructability Reasoning with a Geometrical and Topological Project Model", Computing Systems in Engineering, 4(2-3), Great Britain, 179-192, 1993.

Ikeda, M., Oloufa, A.A., and Sekihara, S. "An Object Oriented Environment for the Construction Planning of High Rise Buildings", Proceedings of the $13^{\text {th }}$ International Symposium on Automation and Robotics in Construction, Tokyo, Japan, 411420, 1996.

Nguyen, T.H.: "Automated Deduction of Topological Information in a Computer-Based Building Design System", Ph.D. Dissertation at Department of Architectural Engineering at the Pennsylvania State University, University Park, PA, U.S.A, 1999. 
Shaked, O. and Warszawski, A.: "KnowledgeBased System for Construction Planning of High-Rise Buildings", Journal of Construction Engineering and Management, ASCE, 21(2), 172-182, 1995.

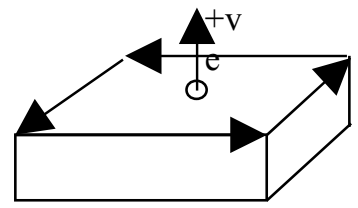

Figure 1. Convention for Face Outward Normal
ObjectARX: "ObjectARX Software Development Kit for AutoCAD Release 14", Autodesk Inc., U.S.A, 1998.

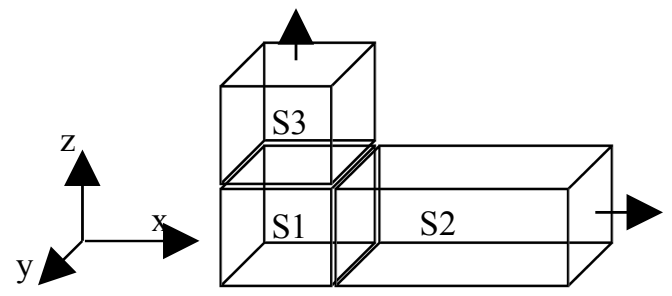

Figure 2. 3D Models of Building Objects

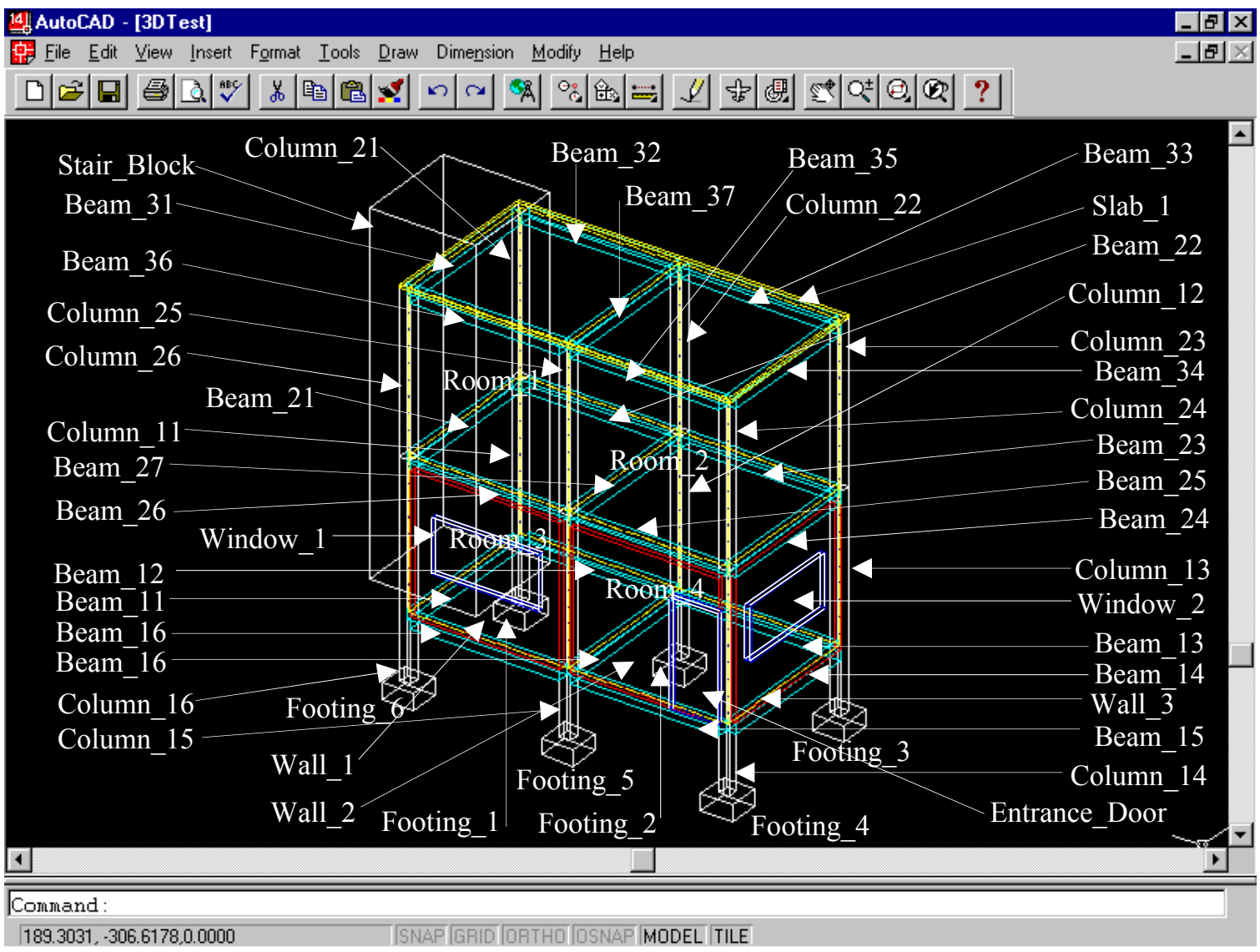

Figure 3. 3D Model of a Typical Building 